\title{
Ground-state degeneracy for Abelian anyons in the presence of gapped boundaries
}

\author{
Anton Kapustin \\ California Institute of Technology, Pasadena, California 91125, USA
}

(Received 24 July 2013; revised manuscript received 21 February 2014; published 19 March 2014)

\begin{abstract}
Gapped phases with long-range entanglement may admit gapped boundaries. If the boundary is gapped, the ground-state degeneracy is well defined and can be computed using methods of topological quantum field theory. We derive a general formula for the ground-state degeneracy for Abelian fractional quantum Hall phases, including the cases when connected components of the boundary are subdivided into an arbitrary number of segments, with a different boundary condition on each segment, and in the presence of an arbitrary number of boundary domain walls.
\end{abstract}

DOI: 10.1103/PhysRevB.89.125307

PACS number(s): 71.10.Pm, 03.65.Vf, 03.70.+k, 73.43.-f

\section{INTRODUCTION}

The simplest fractional quantum Hall phases with $v=1 / p$ are characterized by the presence of chiral gapless edge modes [1,2]. Being chiral, these modes cannot be gapped by any boundary perturbation. However, more general FQH states may have nonchiral edge states which can be lifted. In such cases both the bulk and the boundary are gapped, and one may pose the question of computing the ground-state degeneracy of such a system. A macroscopic quantum degeneracy would be of particular interest if it occurred in a system with a simple topology, such as a disk or an annulus, since such a system would be easier to realize experimentally.

The simplest possibility is to impose the same boundary condition along each connected component of the boundary. One may also place quasiparticles both in the bulk and on the boundary. However, one can show that for Abelian systems this does not lead to quantum degeneracy unless the geometry is nonplanar (and thus hard to realize in practice). More generally, one can consider the situation when each connected component has several segments, with different boundary conditions on each segment and with boundary domain walls separating the segments. A boundary domain wall may be regarded as a quasiparticle sitting at the junction of two different kinds of gapped boundaries. We will see that using boundary domain walls it is possible to create a quantum ground-state degeneracy even in a very simple geometry, like a disk. Examples of this kind were recently given in $[3,4]$. Our results go beyond those in $[3,4]$ in several respects. The method of $[3,4]$ was geared towards a rather special case where the geometry is that of a polygon with an even number of sides, only two boundary conditions $M$ and $M^{\prime}$ are involved, and they are imposed in an alternating manner: $M M^{\prime} M M^{\prime} \ldots$ We consider the case of a polygon with an arbitrary number of sides and with an arbitrary set of boundary conditions imposed. (In fact, we also explain how to compute the degeneracy in the case when the geometry is that of a genus- $g$ Riemann surface with $h$ holes, but this is of less practical interest.) Second, while the method of [3,4] was based on identifying a noncommutative operator algebra acting on the space of ground states and then studying its irreducible representations, we give a closed formula for the number of ground states.

Macroscopic properties of Abelian FQH phases are described by Abelian Chern-Simons theory [1,2] which is a three-dimensional topological quantum field theory (3d TQFT). Gapped boundary conditions correspond to topological boundary conditions in TQFT; for Abelian ChernSimons theory they have been studied in [5-7] while a more general theory based on fusion categories was developed in $[8,9]$. The problem of computing the ground-state degeneracy was previously addressed in [10], but only in the case when there are no boundary domain walls. Our approach is based on reducing the problem to a problem in $2 \mathrm{~d}$ TQFT which can then be analyzed using fairly standard methods.

\section{ABELIAN CHERN-SIMONS THEORY}

\section{A. Bulk properties}

This section is a review of Abelian Chern-Simons theory, including topological boundary conditions, following [5,7].

Consider a general Abelian Chern-Simons gauge theory with gauge group $T \simeq U(1)^{N}$ [1]. The fields in this theory are $U(1)$ gauge fields $A^{i}=A_{\mu}^{i} d x^{\mu}$, where $i=$ $1, \ldots, N$ and $\mu=0,1,2$. They have the usual gaugeinvariance

$$
A_{\mu}^{i} \mapsto A_{\mu}^{i}+\partial_{\mu} f^{i}
$$

where $f^{i}$ are arbitrary real functions which are allowed to be multivalued, $f^{i} \sim f^{i}+2 \pi$. The action is

$$
S=\frac{K_{i j}}{4 \pi} \int_{M} \epsilon^{\mu \nu \rho} A_{\mu}^{i} \partial_{\nu} A_{\rho}^{j} d^{3} x .
$$

To ensure the invariance of $\exp (2 \pi i S)$ under arbitrary gauge transformations, the symmetric matrix $K$ must be integral. For the theory to be well-defined, $K$ must also be non-degenerate. Naively, since the action does not involve the metric, it defines a TQFT. It turns out that on the quantum level this is true only if the diagonal entries of $K$ are even; otherwise the theory also depends on the choice of spin structure on $M[11,12]$. For our purposes this distinction will be unimportant, since we will be mostly interested in topologically trivial spaces.

FQH effect (FQHE) quasiparticles are represented by Wilson loops:

$$
\exp \left(i \oint Q_{i} A_{\mu}^{i} d x^{\mu}\right)
$$

Here $Q_{i}$ is the electric charge with respect to the $i$ th component of the gauge field. Since the gauge group is compact, $Q_{i}$ is an integer. There are also monopole instantons. In Chern-Simons 
theory, a monopole with magnetic charge $\Lambda^{i}$ can absorb a quasiparticle of charge $Q_{i}=K_{i j} \Lambda^{j}$. Thus the electric charge is conserved only modulo vectors of the form $K_{i j} \Lambda^{j}$, where the vector $\Lambda^{j}$ is integral. Since $K$ is nondegenerate, such vectors form a finite-index subgroup in the lattice of all charges. The quotient group is a finite group which we will denote D. Its elements label quasiparticles which cannot disappear into the vacuum.

Another way to explain why quasiparticles are labeled by integer vectors $Q_{i}$ modulo the equivalence $X_{i} \sim X_{i}+K_{i j} \Lambda^{j}$ is to note that a quasiparticle with charge $Q_{j}$ creates a vortex with holonomy

$$
\phi^{i}=\oint A^{i}=2 \pi\left(K^{-1}\right)^{i j} Q_{j}, \quad j=1, \ldots, N .
$$

Since $\phi^{i}$ is periodically identified with period $2 \pi$, shifting $Q_{i}$ by $K_{i j} \Lambda^{j}$ gives an equivalent holonomy. If a quasiparticle is characterized by the holonomy it creates, then one has to identify $Q_{i}$ and $Q_{i}+K_{i j} \Lambda^{j}$.

Taking a quasiparticle with charge $Q_{i}^{\prime}$ around a quasiparticle with charge $Q_{i}$ multiplies the wave function by a phase

$$
\exp \left(2 \pi i Q_{i}^{\prime}\left(K^{-1}\right)^{i j} Q_{j}\right)=\exp \left(\frac{i}{2 \pi} \phi^{\prime i} K_{i j} \phi^{j}\right) .
$$

The braiding phase is unchanged if one replaces the charge vector $Q$ or $Q^{\prime}$ by an equivalent one.

In many respects Abelian Chern-Simons theory resembles a $3 d$ gauge theory with a finite Abelian gauge group $G$. In the latter theory, there are both "vortex" quasiparticles and electric quasiparticles [13]. The former are labeled by their holonomies which are elements of $G$, while the latter are labeled by their charges which are elements of the Pontryagin-dual group $G^{*}$. By definition, an element of $G^{*}$ is the character of an irreducible representation of $G$.

Chern-Simons theory is very similar, except that electrically charged quasiparticles are also vortices, so the two kinds of quasiparticles are identified. The effective gauge group $G$ can be identified with the subgroup of $U(1)^{N}$ spanned by the possible holonomies of electric quasiparticles., i.e., $G$ is the subgroup consisting of elements of the form (4). Equivalently, $G$ can be described as a subgroup of $U(1)^{N}$ consisting of phase vectors $\Phi=\phi^{1}, \ldots, \phi^{N}$ such that

$$
\exp \left(i \phi^{i} K_{i j} \Lambda^{j}\right)=1
$$

for any integer vector $\Lambda^{j}$. We will see in the next section that after compactification to $2 \mathrm{~d}$ Abelian Chern-Simons theory becomes ordinary $2 \mathrm{~d}$ gauge theory with gauge group $G$. Note that the finite group $D$ whose elements label quasiparticles can be thought of as the group of characters of $G$ (i.e., the Pontryagin dual $G^{*}$ of $G$ ). Indeed, given an integral vector $Q_{i}$ we can associate to it a character $\exp \left(i \phi^{i} Q_{i}\right)$. This character depends only on the equivalence class of $Q$, and all characters of $G$ have this form.

\section{B. Boundary properties}

Elementary topological boundary conditions correspond to special subgroups of D called Lagrangian subgroups [5,7]. By definition, a Lagrangian subgroup $L \subset D$ consists of charges of quasiparticles which satisfy the following two properties:

(i) The braiding phase of any two quasiparticles in $L$ is trivial.

(ii) $L$ is maximal, in the sense that any quasiparticle not in $L$ has a nontrivial braiding with at least one quasiparticle in $L$.

The physical meaning of $L$ is this: its elements label those bulk quasiparticles whose charges can be screened once brought to the boundary. One can interpret this as the presence of a condensate of quasiparticles with charges in $L$.

An equivalent way to characterize a boundary condition is to ask which subgroup of the bulk gauge group is unbroken on the boundary. An element $\Phi \in G$ belongs to the unbroken subgroup $H$ only if and only if all quasiparticles in $\mathrm{L}$ have zero charge with respect to $\Phi$, that is, $\exp \left(i \phi^{i} Q_{i}\right)=1$ for all $Q \in \mathrm{L}$. This implies that $H$ consists of elements of the form $\phi^{i}=2 \pi\left(K^{-1}\right)^{i j} Q_{j}$, where $Q_{j}$ is an arbitrary vector in $\mathrm{L}$. The Lagrangian condition can be equivalently formulated in terms of $H \subset G$ instead of $\mathrm{L} \subset \mathrm{D}$, since the braiding phase (5) can equally well be expressed in terms of the holonomy vector $\phi^{i}$.

A Lagrangian subgroup always has order which is the square root of the order of D [7]. Indeed, consider again the map which sends a charge vector $X \in \mathrm{D}$ to the corresponding group element in $G, \phi^{i}=2 \pi\left(K^{-1}\right)^{i j} X_{j}$. This map is an isomorphism between $\mathrm{D}$ and $G$. Since $G$ is the Pontryagin dual of $D$, we get an isomorphism of $D$ and $D^{*}$. Now let $L$ be a Lagrangian subgroup of $D$. The above map sends $L$ to a character of $D$ which is trivial on $L$, by virtue of the first part of the Lagrangian condition. That is, it maps $L$ to $(D / L)^{*}$. The second part of the Lagrangian condition implies that this map is an isomorphism. Let $|G|$ denote the order of a finite group $G$. Since $|\mathrm{D} / \mathrm{L}|=|\mathrm{D}| /|\mathrm{L}|$, we conclude that $|\mathrm{L}|^{2}=|\mathrm{D}|$. Thus a necessary condition for the existence of topological boundary conditions is that the number of distinct bulk quasiparticles is a square of an integer. The order of the unbroken gauge group $H$ is the same as the order of L, i.e., $\sqrt{|\mathrm{D}|}=\sqrt{|G|}$.

A point where two segments of the boundary meet will be called a boundary domain wall. The boundary conditions on the two segments can be the same or different. Boundary domain walls may be characterized by the electric charges they carry, or equivalently by the holonomy of the $U(1)^{N}$ gauge field along a small semi-circle enclosing the domain wall. If we take the former viewpoint, then elementary boundary domain walls correspond to integer vectors $Q_{i}$ modulo elements of the form $K_{i j} \Lambda^{j}+L_{i}+L_{j}^{\prime}$, where $\Lambda^{i}$ is an arbitrary integer vector, as before, and $L_{i}$ and $L_{i}^{\prime}$ are arbitrary charge vectors which lie in the subgroups $L$ and $L^{\prime}$, respectively [5]. Indeed, at the boundary domain wall both condensates with charges in $L$ and $\mathrm{L}^{\prime}$ are present, and boundary domain walls which differ only by the emission or absorption of the condensate particles are indistinguishable.

Alternatively, we can associate to a quasiparticle with charge $Q_{i}$ the corresponding holonomy (4) in the effective gauge group $G$. Since the charge of the boundary domain wall is defined only modulo elements in $L$ and $L^{\prime}$, the corresponding holonomy is defined only modulo elements in the subgroups $H$ and $H^{\prime}$ corresponding to $\mathrm{L}$ and $\mathrm{L}^{\prime}$. In what follows we will mostly use this alternative viewpoint and label an elementary boundary domain wall by elements of $G$ defined modulo 
elements of $H$ and $H^{\prime}$. In mathematics, this is known as the set of cosets of $G$ with respect to the subgroup $H+H^{\prime}$.

We are interested in the situation when the spatial geometry is that of a compact oriented 2-manifold $\Sigma$ with a non-empty boundary. Each boundary component $\Gamma_{a}$ is a circle and is subdivided into segments $\Gamma_{a}^{1}, \ldots, \Gamma_{a}^{N_{a}}$. On a segment $\Gamma_{a}^{i}$ one picks a Lagrangian subgroup $L_{a}^{i} \subset \mathrm{D}$, or equivalently a Lagrangian subgroup $H_{a}^{i} \subset G$, while at the junction of two consecutive segments $\Gamma_{a}^{i}, \Gamma_{a}^{i+1}$ one picks an element of $G /\left(H_{a}^{i}+H_{a}^{i+1}\right)$. We are going to compute the dimension of the space of states $\mathcal{H}$ of Abelian Chern-Simons theory in this situation. It is equal to the ground-state degeneracy of the corresponding FQHE system.

\section{REDUCTION TO TWO DIMENSIONS}

\section{A. From 3d Chern-Simons to 2d gauge theory}

Consider the path integral for $3 \mathrm{~d}$ Chern-Simons theory on $\Sigma \times S^{1}$, where $\Sigma$ is a 2 d manifold with a boundary. Its partition function is equal to the dimension of the vector space $\mathcal{H}_{\Sigma}$. Hence we can rephrase the problem as follows. Consider the $2 \mathrm{~d}$ TQFT obtained by compactifying the Chern-Simons theory on $S^{1}$. Each topological boundary condition reduces to a topological boundary condition in this $2 \mathrm{~d}$ TQFT. Boundary domain walls in $3 \mathrm{~d}$ reduce to particular boundary-changing operators in the $2 \mathrm{~d}$ TQFT. The dimension of $\mathcal{H}_{\Sigma}$ is therefore equal to the $2 \mathrm{~d}$ topological correlator on $\Sigma$.

Let us identify the $2 \mathrm{~d}$ TQFT that one obtains by compactification on a circle. Each $3 \mathrm{~d}$ gauge field $A^{i}$ gives rise to a periodic scalar $\phi^{i} \sim \phi^{i}+2 \pi$. This scalar is the holonomy of $A^{i}$ along $S^{1}$, and periodic identification arises from gauge transformations which have a nontrivial winding number on $S^{1}$. The components of $A^{i}$ along $\Sigma$ can be regarded as a $2 \mathrm{~d}$ gauge field which we denote by the same letter. On the classical level, compactification is very simple: one simply considers configurations of $3 \mathrm{~d}$ gauge fields which are independent of the circle coordinate and evaluates the action on such configurations. The resulting $2 \mathrm{~d}$ action is

$$
S_{2}=\frac{1}{2 \pi} \int_{\Sigma} K_{i j} \epsilon^{\mu v} \phi^{i} \partial_{\mu} A_{\nu}^{j} .
$$

This action describes a topological gauge theory with a finite Abelian gauge group $G \subset U(1)^{N}$. To see this, let us dualize the scalars $\phi^{i}$. To this end we note that $\phi^{i}$ enters only through its derivatives $h_{\mu}^{i}=\partial_{\mu} \phi^{i}$. The vector field $h_{\mu}^{i}$ has a vanishing curl, so we can trade $\phi^{i}$ for $h_{\mu}^{i}$ and a Lagrange multiplier field $\chi_{i}$ whose equation of motion enforces the vanishing of the curl of $h_{\mu}^{i}$. This leads to the following equivalent action:

$$
S_{2}^{\prime}=-\int_{\Sigma} \epsilon^{\mu \nu}\left(\partial_{\mu} \chi_{i}-K_{i j} A_{\mu}^{j}\right) h_{\nu}^{i} .
$$

There is a slight subtlety in that the vector field $h_{\mu}^{i}$ must satisfy an additional condition: its integral along any closed curve must be an integer multiple of $2 \pi$. One can show that this condition is enforced if $\chi^{i}$ is periodically identified with period $2 \pi$, and one includes in the path-integral sectors with all possible winding numbers for $\chi^{i}$ (see, e.g., $[14,15]$ ).
The fields transform under gauge transformations as follows:

$$
\chi_{i} \mapsto \chi_{i}+K_{i j} \sigma^{j}, \quad A_{\mu}^{i} \mapsto A_{\mu}^{i}+\partial_{\mu} \sigma^{i} .
$$

where the functions $\sigma^{i}$ parametrize the gauge transformation. Locally we can choose the gauge $\chi_{i}=0$. Since $\chi_{i}$ is periodically identified, this does not completely fix the gauge freedom: we are left with constant gauge transformations $\sigma^{i}(x)=\sigma_{0}^{i}$ such that for all $i$ we have

$$
\frac{1}{2 \pi} K_{i j} \sigma_{0}^{j} \in \mathbb{Z}
$$

These are precisely gauge transformations which lie in the subgroup $G$ defined by (6). This means that in the gauge $\chi_{i}=0$ the gauge field is zero locally, but the transition functions must be constant and take values in the subgroup $G$. Such a gauge field is flat, and its holonomy takes values in $G$. Thus the path-integral reduces to a sum over flat $G$ connections. The classical action vanishes on such configurations, so each term in the sum has the same weight.

The weight is a bit subtle, since it requires specifying the measure in the path integral. The usual normalization factor is $1 /|G|[13]$. It is determined as follows. One the one hand, the partition function on a torus $T^{2}$ must be equal to the dimension of the quantum space of states of the $2 \mathrm{~d}$ theory on $S^{1}$. This dimension is $|G|$, since there are exactly $|G|$ distinct flat connections on $S^{1}$, and each of them gives rise to a single state in the quantum theory. On the other hand, the number of flat connections on $T^{2}$ is $|G|^{2}$, hence we need a normalization factor $1 /|G|$.

However, this argument neglects the possibility that the path-integral measure might depend on the geometry of $\Sigma$. Such dependence can be thought of as a local geometric term in the action, i.e., a term which does not depend on the fields but depend on the topology of $\Sigma$. In $3 \mathrm{~d}$, there are no such terms, but in $2 \mathrm{~d}$ we can add to the action a geometric term

$$
\frac{\mu}{2 \pi} \int_{\Sigma} R \sqrt{g} d^{2} x=\mu \chi(\Sigma),
$$

where $\chi(\Sigma)=2-2 g$ is the Euler characteristic of $\Sigma$. Such a term modifies the partition function by a factor $e^{\mu(2-2 g)}$ and therefore does not contribute for $\Sigma=T^{2}$. To determine $\mu$, we need to consider nonflat $\Sigma$. It is well known that the number of states of the Abelian Chern-Simons theory on a genus- $g$ Riemann surface without boundary is $\mid G^{g}$ [2]. On the other hand, with the usual normalization factor $1 /|G|$ the partition function of the $2 \mathrm{~d}$ topological gauge theory is the number of flat $G$ connections on $\Sigma$ divided by $|G|$, i.e., $|G|^{2 g-1}$. They agree for $g=1$ but disagree for other $g$. This implies that the dimensional reduction of Chern-Simons theory gives a $2 \mathrm{~d}$ gauge theory with a geometric term (11) and $e^{\mu}=\sqrt{|G|}$.

\section{B. Bulk properties of the $2 \mathrm{~d}$ gauge theory}

The bulk properties of the $2 d$ gauge theory with a discrete Abelian gauge group $G$ are very simple. Classical configurations on a circle are labeled by the holonomy of the discrete gauge field, therefore the quantum space of states has dimension $|G|$. It has a distinguished basis $e_{g}, g \in G$, where $e_{g}$ is the quantum state corresponding to the holonomy $g$. Axioms 
of $2 \mathrm{~d}$ TQFT say that there is a 1-1 correspondence between states and local operator insertions $[16,17]$. By definition, a local operator insertion corresponding to $e_{g}$ creates a singularity in the gauge field with holonomy $g$. Axioms of TQFT also tell us that local operators form a commutative and associative algebra with respect to the usual operator product. Clearly, fusing local operators $e_{g}$ and $e_{g^{\prime}}$ gives a singularity with gauge field holonomy $g+g^{\prime}$. Hence the product of $e_{g}$ and $e_{g^{\prime}}$ is proportional to $e_{g+g^{\prime}}$. Since the algebra of bulk local operators is commutative, the multiplication law must have the form

$$
e_{g} \circ e_{g^{\prime}}=f\left(g, g^{\prime}\right) e_{g+g^{\prime}},
$$

where $f\left(g, g^{\prime}\right)$ is a function on $G \times G$ satisfying $f\left(g, g^{\prime}\right)=$ $f\left(g^{\prime}, g\right)$. It is assumed that $f\left(g, g^{\prime}\right) \neq 0$ for all $g, g^{\prime}$. Associativity of the operator product implies that $f\left(g, g^{\prime}\right)$ satisfies the cocycle condition

$$
f\left(g+g^{\prime}, g^{\prime \prime}\right) f\left(g, g^{\prime}\right)=f\left(g, g^{\prime}+g^{\prime \prime}\right) f\left(g^{\prime}, g^{\prime \prime}\right) .
$$

Thus $f$ is a 2-cocycle on $G$ with values in the group of nonzero complex numbers. It follows from the universal coefficients formula that any such cocycle is a coboundary [18], that is, there exists a function $h(g)$ on $G$ such that

$$
f\left(g, g^{\prime}\right)=h(g) h\left(g^{\prime}\right) h\left(g+g^{\prime}\right)^{-1} .
$$

This means that by rescaling $e_{g} \rightarrow h(g) e_{g}$ one can remove $f\left(g, g^{\prime}\right)$ so that the multiplication law becomes

$$
e_{g} \circ e_{g^{\prime}}=e_{g+g^{\prime}} \text {. }
$$

This rule completely describes the algebra of bulk local operators. This algebra is known as the group algebra of $G$.

An equivalent way to describe this algebra is to note that the most general local operator $\sum_{g} c(g) e_{g}, c(g) \in \mathbb{C}$, depends on a complex-valued function $c(g)$ on $G$. Thus one can identify the space of local operators with the space of such functions. It is easy to see that the multiplication rule (15) for the basis elements $e_{g}$ is equivalent to the following rule for the multiplication of functions:

$$
\left(c_{1} \circ c_{2}\right)(g)=\sum_{g^{\prime} \in G} c_{1}\left(g^{\prime}\right) c_{2}\left(g-g^{\prime}\right) .
$$

That is, the product is given by the convolution of functions. Note that the local operator $e_{g_{0}}$ corresponds to a function $c(g)$ which takes value 1 on $g_{0}$ and is zero everywhere else. We will denote such function $\delta\left(g-g_{0}\right)$.

The local operator $e_{g}$ can be thought of as the dimensional reduction of a quasiparticle in the $3 \mathrm{~d}$ theory whose wordline is a loop wrapping $S^{1}$. Indeed, $e_{g}$ is a prescription to perform a path integral over flat gauge fields whose holonomy around the insertion point is $g$. Since a quasiparticle in $3 \mathrm{~d}$ creates precisely such a gauge field, its dimensional reduction must be identified with $e_{g}$, at least up to a numerical factor.

We can fix this normalization factor by computing the $2 \mathrm{~d}$ partition function on $S^{2}$ with two operator insertions $e_{g_{1}}$ and $e_{g_{2}}$. From the $3 \mathrm{~d}$ viewpoint, this must be the dimension of the state space of Abelian Chern-Simons theory on $S^{2}$ with two quasiparticles with holonomies $g_{1}$ and $g_{2}$. Therefore we expect to get $\delta\left(g_{1}+g_{2}\right)$ (if the holonomies do not add up to zero, the space is zero dimensional, otherwise it is one-dimensional because the two quasiparticles can fuse together and annihilate each other). The $2 \mathrm{~d}$ path integral, including the normalization factor $e^{\mu \chi}$, is

$$
Z\left(g_{1}, g_{2}\right)=\frac{(\sqrt{|G|})^{2}}{|G|} \delta\left(g_{1}+g_{2}\right)=\delta\left(g_{1}+g_{2}\right),
$$

since there is a unique flat connection with prescribed singularities if $g_{1}+g_{2}=0$ and no flat connections otherwise. Thus the operator $e_{g}$ is precisely the compactification of an elementary quasiparticle in $3 \mathrm{~d}$, without the need for any additional factors.

While in the case of discrete gauge theory one can perform the sum over flat connections in the continuum, one can also use a lattice model. To describe this model, let us foliow Dijkgraaf and Witten [13] and pick a triangulation of $\Sigma$, or better yet, a cell decomposition of $\Sigma$, since this allows more flexibility. A cell decomposition of $\Sigma$ is a graph whose complement consists of several regions (2-cells). Edges of a graph will be referred to as 1-cells, while vertices will be referred to as 0-cells. We assume that each 1-cell has been oriented in some way and place a variable $g \in G$ on each oriented 1-cell, so that reversing orientation changes $g \mapsto-g$. These variables must satisfy the flatness constraint which says that for any 2-cell $P$ the signed sum of variables corresponding to all 1-cells in the closure of $P$ vanishes. The constraint ensure the absence of "lattice vortices." The partition function is the sum over all choices of 1-cell variables satisfying the flatness constraints, divided by the order of the gauge group. Gauge transformations are $G$-valued functions on 0-cells, acting on 1 -cell variables in an obvious manner. Finally, an insertion of a local operator $e_{g}$ corresponds to modifying the flatness prescription for a particular 2-cell so that 1-cell variables sum to $g$ instead of zero. We will find the lattice formulation useful when we study the partition function in the presence of boundaries and boundary domain walls.

\section{DISK CORRELATORS IN THE 2D TQFT}

Topological boundary conditions in the topological $2 \mathrm{~d}$ gauge theory with gauge group $G$ are labeled by representations of $G$. A boundary condition corresponding to a representation $V$ is equivalent to placing a charged particle in the representation $V$ on the boundary. In other words, for every boundary component labeled by $V$ one inserts a Wilson loop in the representation $V$. Elementary boundary conditions correspond to irreducible representations of $G$. It is also easy to describe boundary-changing operators: they are maps between representations which commute with the action of $G$. However, not all $2 \mathrm{~d}$ boundary conditions arise by dimensional reduction from $3 \mathrm{~d}$ boundary conditions, and moreover an elementary $3 \mathrm{~d}$ boundary condition after compactification on a circle becomes a sum of elementary $2 \mathrm{~d}$ boundary conditions. For this reason we focus on a special class of $2 \mathrm{~d}$ boundary conditions labeled by subgroups of $G$. Namely, for every subgroup $H \subset G$ we may consider a boundary where $G$ is broken down to $H$, and no additional weights in the path integral. This can be achieved by constraining both $A$ and $\chi$ on the boundary. From a representation-theoretic perspective, such a boundary condition corresponds to a representation of $G$ in the space of 
functions on $G / H$. In general it is a reducible representation. In the Abelian case that we are discussing, the subgroup $H$ acts trivially on such functions, while the rest of the group acts nontrivially. Thus a particle in such a representation breaks the gauge group down to $H$.

We focus on these $2 \mathrm{~d}$ boundary conditions because they are more naturally related to $3 \mathrm{~d}$ boundary conditions. Namely, as explained in Sec. II B, a boundary condition in Abelian ChernSimons theory is completely determined by the unbroken gauge group on the boundary. Note that the converse is not true: a 2 d boundary condition corresponding to a subgroup $H$ can be lifted to a $3 \mathrm{~d}$ boundary condition only if $H$ is a Lagrangian subgroup of $G$. Nevertheless, since in the $2 \mathrm{~d}$ computations the Lagrangian condition does not play a role, we may leave $H$ arbitrary for now.

Consider now an oriented surface $\Sigma$ with a nonempty boundary. Each boundary component is a circle and may be subdivided into segments so that there is a different boundary condition on each segment. At each junction of two boundary segments there is a boundary-changing operator. In the case of interest for us these boundary-changing operators arise from the boundary domain walls in the $3 \mathrm{~d}$ theory, but this fact is not important for the time being. Using the factorization properties of $2 \mathrm{~d}$ TQFT $[16,17]$, one can replace each boundary component with a local operator. This local operator can be expanded in our preferred basis $\left\{e_{g}\right\}$, and the expansion coefficients are equal to the disk correlator with an insertion of $e_{g}$ in the bulk. Thus our problem is reduced to evaluating such disk correlators in the $2 \mathrm{~d}$ TQFT.

As a preliminary step, we need to understand the $2 \mathrm{~d}$ interpretation of boundary domain walls. As explained in Sec. II B, given two boundary conditions labeled by subgroups $H_{1}$ and $H_{2}$, the set of boundary domain walls is $G /\left(H_{1}+H_{2}\right)$. A boundary domain wall labeled by $[g] \in G /\left(H_{1}+H_{2}\right)$ has a singularity in the gauge field with holonomy $g$. The holonomy vector $g^{i}=\int A_{\mu}^{i} d x^{\mu}$ is defined only modulo elements in $H_{1}+$ $\mathrm{H}_{2}$ because the holonomy is measured along a semi-circle, rather than a circle. Thus boundary gauge transformations will shift $g$ by elements in $H_{1}+H_{2}$ :

$$
g \mapsto g+h_{2}-h_{1}, \quad h_{1} \in H_{1}, \quad h_{2} \in H_{2} .
$$

Nevertheless, since $H_{1}+H_{2}$ need not be the whole $G$, some gauge-invariant information survives.

Upon reduction to $2 \mathrm{~d}$, each boundary domain wall gives rise to a boundary-changing operator. Let us denote the boundarychanging operator corresponding to $x \in G /\left(H_{1}+H_{2}\right)$ by $\psi_{x}$. It turns out that any boundary-changing operator is a linear combination of $\psi_{x}$ for different possible $x$. If we think about $\psi_{x}$ as a function on $G /\left(H_{1}+H_{2}\right)$ which is equal to 1 on $x$ and zero elsewhere, then this is equivalent to saying that the space of boundary-changing operators is the space of complex-valued functions on $G /\left(H_{1}+H_{2}\right)$. Indeed, axioms of 2d TQFT [17] tell us that the former space can be identified with the space of states of the TQFT on an interval, with the boundary conditions corresponding to $H_{1}$ and $H_{2}$ on the two ends. This is a boundary version of the state-operator correspondence familiar from $2 \mathrm{~d}$ conformal field theory. Quantization of $2 \mathrm{~d}$ topological gauge theory on an interval is very simple: classical configurations are labeled by the holonomy of $G$ modulo gauge transformations, that is, by elements of $G /\left(H_{1}+H_{2}\right)$,

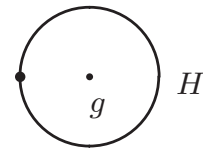

(a)

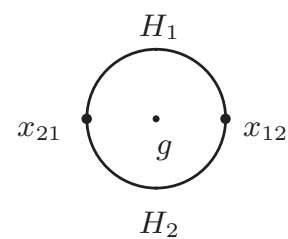

(b)
FIG. 1. (a) A cellular decomposition of a disk, with an insertion of a local operator $e_{g}$ in the interior of the 2-cell. (b) A cellular decomposition of a disk, with an insertion of a local operator $e_{g}$ in the interior of the 2-cell and two boundary-changing operators.

and each classical configuration gives rise to a quantum state. Hence the quantum space of states is the space of functions on $G /\left(H_{1}+H_{2}\right)$. It is easy to see that the dimension of the space of boundary-changing operators is

$$
\frac{|G|}{\left|H_{1}+H_{2}\right|}=\frac{|G|\left|H_{1} \cap H_{2}\right|}{\left|H_{1}\right|\left|H_{2}\right|} \text {. }
$$

It is convenient to regard a function on $G /\left(H_{1}+H_{2}\right)$ as a function on $G$ which is invariant under translations by elements of $H_{1}$ and $H_{2}$. Then one has

$$
\psi_{x}(g)=\sum_{g \in G,[g]=x} e_{g}, \quad x \in G /\left(H_{1}+H_{2}\right) .
$$

The above arguments imply that the dimensional reduction of a boundary domain wall labeled by $x \in G /\left(H_{1}+H_{2}\right)$ is given by $\psi_{x}(g)$, up to a normalization factor. The normalization factor will be fixed below.

To evaluate disk correlators, let us begin with the case when there are no boundary-changing operators, and accordingly the whole boundary is labeled by a subgroup $H$. As usual, the partition function can be evaluated by summing over all flat connections and dividing by the order of the gauge group. The only difference compared to the no-boundary case is that variables living on 1-cells which lie on the boundary take values in $H$, rather than in $G$, and the gauge transformations at the boundary 0-cells also lie in $H$. We also need to multiply the partition function by the factor $e^{\mu}=\sqrt{|G|}$, since the Euler characteristic of the disk is 1 . The simplest cell decomposition of the disk depicted in Fig. 1(a) yields the following expression for the partition function:

$$
Z_{1 a}=\frac{\sqrt{|G|}}{|H|} \delta(g) .
$$

If $H$ corresponds to a Lagrangian subgroup in $\mathrm{D}$, this is equal to $\delta(g)$, i.e., the $3 \mathrm{~d}$ space of states is one-dimensional if $g=0$ and zero otherwise. This is the expected result, confirming that the partition function was normalized correctly.

Next we consider the case of a disk with two boundary segments labeled by subgroups $H_{1}$ and $H_{2}$, a bulk insertion $e_{g}$ and two boundary-changing operators which are obtained from by dimensional reduction from boundary domain walls in $3 \mathrm{~d}$ [see Fig. 1(b)]. As explained above, a boundary domain wall labeled by $x \in G /\left(H_{1}+H_{2}\right)$ reduces to a $\psi_{x}$, up to a normalization factor. To fix the normalization factor, let us compute the partition function corresponding to Fig. 1(b). 


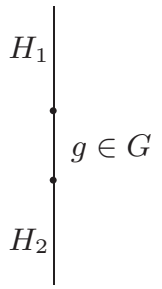

FIG. 2. In the lattice model, a boundary-changing operator $\psi_{x}$ corresponds to a special 1-cell separating two segments of the boundary. One sums over all values of the variable $g \in G$ living on this 1-cell such that $g$ corresponds to a fixed $x \in G /\left(H_{1}+H_{2}\right)$.

Following the above rules and taking into account only the usual normalization factor $\sqrt{|G|}$, we find

$$
Z_{1 b}=\frac{\sqrt{|G|}}{\left|H_{1} \cap H_{2}\right|} \delta\left([g]+x_{12}+x_{21}\right)
$$

In particular, for $g=0$ and $x_{12}=-x_{21}$ the disk partition function is equal to $\sqrt{|G|} /\left|H_{1} \cap H_{2}\right|$.

On the other hand, the $3 \mathrm{~d}$ version of the state-operator correspondence (see, e.g., [19]) implies that for $g=0$ the space of states of the Abelian Chern-Simons theory corresponding to Fig. 1(b) can be identified with the space of local operators inserted at the boundary domain wall corresponding to $x_{12}$. This boundary domain wall is simply a Wilson line (i.e., a charged quasiparticle) at the junction of two boundary conditions. It has no internal degrees of freedom, therefore there are no nontrivial local operators which can be inserted there. (This is equivalent to saying that the boundary domain wall is elementary.) Thus we expect $Z_{1 b}=1$. To achieve this, the dimensional reduction of the elementary boundary domain wall must be $\psi_{x}$ times the normalization factor

$$
c_{12}=\sqrt{\frac{\left|H_{1} \cap H_{2}\right|}{\sqrt{|G|}}} .
$$

Note that this factor is 1 in the special case when $H_{1}=H_{2}$ and $\left|H_{1}\right|=\sqrt{|G|}$.

We are now ready to compute the partition function on a disk for an arbitrary number of boundary segments and arbitrary boundary-changing operators. Boundary segments are labeled by subgroups $H_{1}, \ldots, H_{N}$, while boundary-changing operators are labeled by elements $x_{i} \in G /\left(H_{i}+H_{i+1}\right), i=1, \ldots, N$ with the convention $H_{N+1}=H_{1}$. Including the normalization factors explained above, we get

$$
\begin{aligned}
Z(x ; g)= & (\sqrt{|G|})^{1-N / 2} \prod_{i=1}^{N} \frac{\sqrt{\left|H_{i} \cap H_{i+1}\right|}}{\left|H_{i}\right|} \\
& \times \sum_{\substack{g_{i} \in G \\
\left[g_{i}\right]=x_{i}}} \delta\left(g-\sum_{i=1}^{N} g_{i}\right) .
\end{aligned}
$$

In the case of interest to us, $\left|H_{i}\right|=\sqrt{|G|}$ for all $i$, so we can simplify this a bit to

$$
\begin{aligned}
Z(x ; g)= & (\sqrt{|G|})^{1-3 N / 2} \prod_{i=1}^{N} \sqrt{\left|H_{i} \cap H_{i+1}\right|} \\
& \times \sum_{\substack{g_{i} \in G \\
\left[g_{i}\right]=x_{i}}} \delta\left(g-\sum_{i=1}^{N} g_{i}\right) .
\end{aligned}
$$

It is not at all obvious that this expression is an integer for all conceivable Lagrangian subgroups. We will check this in a few examples below.

\section{EXAMPLES}

Let us compute the ground-state degeneracy in several special cases. First, if $H_{1}=\cdots=H_{N}=H$, we get

$$
Z(x ; g)=\delta\left([g]-\sum_{i} x_{i}\right) .
$$

Here $[g] \in G / H$ is the equivalence class of $g \in G$. Thus if only a single elementary boundary condition is involved, the state space is at most one-dimensional, regardless of the choice of boundary domains walls.

Another simple but interesting case is when two boundary conditions alternate: $H_{1}$, then $H_{2}$, then $H_{1}$ again, etc. Suppose there is a total of $2 k$ of boundary segments, $k \in \mathbb{N}$, so that all boundary domain walls are labeled by elements of the same set $G /\left(H_{1}+H_{2}\right)$. Then we find

$$
Z(x ; g)=\delta\left([g]_{12}-\sum_{i} x_{i}\right)\left(\frac{\left|H_{1}\right|}{\left|H_{1} \cap H_{2}\right|}\right)^{k-1} .
$$

where $[g]_{12} \in G /\left(H_{1}+H_{2}\right)$ is the equivalence class of $g \in G$. In particular, if $H_{1} \cap H_{2}=0$ (i.e., $H_{1}$ and $H_{2}$ are complementary subgroups of $G$ ), the degeneracy is $\left|H_{1}\right|^{k-1}$, provided all charges cancel. This agrees with the computation in $[3,4]$.

Finally, let us consider the case of three boundary segments. For simplicity, let us consider the case of $U(1) \times U(1)$ ChernSimons theory with

$$
K=\left(\begin{array}{ll}
0 & k \\
k & 0
\end{array}\right)
$$

This TQFT is equivalent to a lattice model known as Kitaev's toric code [20]. In this case $\mathrm{D}=G=\mathbb{Z}_{k} \times \mathbb{Z}_{k}$. Lagrangian subgroups in $G$ can be described as follows. First one picks an integer $m$ which divides $k$. Integers divisible by $k / m$ constitute a subgroup $\mathbb{Z}_{m}$ of $\mathbb{Z}_{k}$. Then it is easy to check that the subgroup of $\mathbb{Z}_{k} \times \mathbb{Z}_{k}$ given by

$$
H_{m}=\left\{\left(n_{1}, n_{2}\right) \mid n_{1}=0 \bmod k / m, n_{2}=0 \bmod m\right\}
$$

is Lagrangian, and therefore gives rise to a gapped boundary condition. Let us now pick three integers $m_{1}, m_{2}, m_{3}$ which divide $k$. They correspond to three boundary conditions labeled by subgroups $H_{m_{i}}$. Elementary boundary domain walls are labeled by

$$
x_{i} \in G /\left(H_{m_{i}}+H_{m_{i+1}}\right), \quad i=1,2,3 .
$$




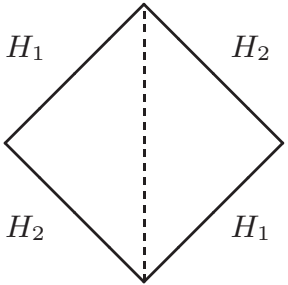

FIG. 3. A nontrivial degeneracy on a triangle can be obtained by starting with a square with an alternating pattern of boundary conditions and folding along the diagonal.

A short computation shows that the disk partition function is

$$
Z(x ; g)=\delta\left(\left[g-x_{1}-x_{2}-x_{3}\right]_{123}\right) .
$$

where $[g]_{123}$ denotes the equivalence class of $g \in G$ in $G /\left(H_{m_{1}}+H_{m_{2}}+H_{m_{3}}\right)$. Thus there is no degeneracy in this case.

One should not think, however, that nontrivial degeneracy is impossible with only three boundary segments. For example, one can take the disk with four boundary segments such there is a nontrivial degeneracy and fold it along a diameter (see Fig. 3).
This gives a theory with a doubled gauge group on a disk with three boundary segments. All boundary conditions and boundary domain wall are elementary, yet there is a nontrivial degeneracy (the same one as before folding).

\section{DISCUSSION}

We have derived a general and easy-to-use formula for the ground-state degeneracy of Abelian FQH systems in the presence of gapped boundaries. It would be interesting to generalize this computation to non-Abelian topological phases in $(2+1) d$ systems. A continuum gauge theory description of such phases it not known, in general, but there exists a Hamiltonian lattice model $[8,21]$. It should be possible to reduce it to $2 \mathrm{~d}$ and compute the partition function in the resulting $2 \mathrm{~d}$ TQFT.

\section{ACKNOWLEDGMENTS}

I would like to thank M. Barkeshli, C. M. Jian, and X. L. Qi for a discussion. This work was supported in part by DOE Grant No. DE-FG02-92ER40701.
[1] X. G. Wen, Adv. Phys. 44, 405 (1995).

[2] X. G. Wen, Quantum Field Theory of Many-Body Systems: From the Origin of Sound to an Origin of Light and Electrons (Oxford University Press, Oxford, 2004).

[3] M. Barkeshli, C. M. Jian, and X. L. Qi, arXiv:1304.7579.

[4] M. Barkeshli, C. M. Jian, and X. L. Qi, arXiv:1305.7203.

[5] A. Kapustin and N. Saulina, Nucl. Phys. B 845, 393 (2011).

[6] A. Kapustin and N. Saulina, in Mathematical Foundations of Quantum Field Theory and Perturbative String Theory, edited by H. Sati and U. Schreiber (American Mathematical Society, Princeton, 2011), pp. 175-198.

[7] M. Levin, Phys. Rev. X 3, 021009 (2013).

[8] A. Kitaev and L. Kong, Commun. Math. Phys. 313, 351 (2012).

[9] J. Fuchs, C. Schweigert, and A. Valentino, arXiv:1203.4568.

[10] J. Wang and X.-G. Wen, arXiv:1212.4863.
[11] E. Witten, Commun. Math. Phys. 121, 351 (1989).

[12] D. Belov and G. W. Moore, arXiv:hep-th/0505235.

[13] R. Dijkgraaf and E. Witten, Commun. Math. Phys. 129, 393 (1990).

[14] E. Witten, Selecta Math. 1, 383 (1995).

[15] A. Kapustin and M. Tikhonov, J. High Energy Phys. 11 (2009) 006.

[16] M. Atiyah, Inst. Hautes Etudes Sci. Publ. Math. 68, 175 (1989).

[17] G. W. Moore and G. Segal, arXiv:hep-th/0609042.

[18] K. Brown, Cohomology of Groups (Springer, Berlin, 1982).

[19] A. Kapustin, in Proceedings of the International Congress of Mathematicians 2010, Hyderabad, Vol. III (World Scientific, Singapore, 2011), pp. 2021-2043.

[20] A. Y. Kitaev, Ann. Phys. (NY) 303, 2 (2003).

[21] M. A. Levin and X. G. Wen, Phys. Rev. B 71, 045110 (2005). 